\title{
COVID - 19 Intubation Box: Is it the New Normal???
}

\author{
Chashamjot Bawa ${ }^{1}$, Rashi Sarna ${ }^{2 *}$, Rajeev Chauhan ${ }^{3}$ \\ ${ }^{1}$ Assistant Professor, Maharishi Markandeshwar Institute of Medical Sciences and Research, Ambala, \\ ${ }^{2,3}$ Assistant Professor, Postgraduate Institute of Medical Education and Research, Chandigarh
}

The ongoing COVID-19 pandemic has thrown a dual challenge before health care workers to treat patients and as well protect themselves. The aerosol generating procedures (AGPs) such as nebulization, bag and mask ventilation, endotracheal intubation carries a maximum risk of viral spread. Most of the above procedures are performed by anaesthesiologists, hence they are recommended to wear highest level of protection.

The advent of 'aerosol box' by Dr. Hsien Yung Lai of Taiwan ${ }^{1}$ has conceptualized the idea of improving safety during high- risk procedures by means of creating an additional barrier between the anaesthesiologist and the patient. Coughsimulation based studies ${ }^{2}$ conducted on the box demonstrated significant benefit in preventing droplet transmission, however many others questioned its role in prevention of viral transmission. Attempts have been made by innovative minds in the anaesthesiology fraternity to overcome some of the inhibitions incurred with this device by structural modifications. However, it is a long way still from reaching the ideal design and usage protocol.

The initial studies suggest prolongation of TimeTo-Intubation (TTI) and increased number of failures with this device ${ }^{3}$; so, there is call for increasing practice with this new equipment, more so in cases of COVID-19 associated

*Correspondence: Rashi Sarna
E mail: sarnarashi@yahoo.com

https://orcid.org/0000-0003-3242-4353

Received: $13 / 09 / 2020$

Accepted: 23/09/2020

DOI: http:/doi.org/10.4038/slja.v29i1.8682

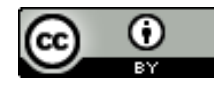

respiratory failure where rapid desaturation can occur. This can only be materialized by augmenting training and research on the subject in the sub-specialties concerned. The national and international organizations and experts in the field must work together by means of webinars to discuss the problems being faced with the box and find solutions. An AGP specific stepwise approach should be undertaken to culminate the current controversies surrounding its use. Randomized controlled trials comparing different modifications and methodology for different AGPs must be encouraged. There is a dire need to generate and share as much knowledge and experience with this equipment as possible to gain expertise.

Furthermore, most nations have decided to live with the unanswered question as to when this pandemic will end and are transitioning from earlier periods of lockdown to resumption of all essential and most non- essential health care services despite the rise in infections. More and more patients are being dealt by anaesthesiologists perioperatively and in ICUs without COVID-19 testing due to the demand supply crisis of testing kits in areas of community transmission. The logical approach with this patient population having unknown COVID-19 status shall be to use PPEs and aerosol boxes while performing AGPs. Is it correct to say in such a scenario that the new post graduates and diploma trainees in anaesthesiology be mannequin trained for AGPs like intubations using PPEs and aerosol boxes right from the start of their curriculum? Is it not the time that these skills be re-learnt with safety precautions and equipment and regularly taught to upcoming batches so that there is quality control and no breech in safety protocols in pandemic era? We are heading towards a future demanding "minimal breath exchange" between patient and anaesthesiologist while airway maneuvering and aerosol boxes being a new normal. 


\section{References}

1. Kearsley R. Intubation boxes for managing the airway in patients with COVID-19. Anaesthesia. 2020; 75(7): 969.

https://doi.org/10.1111/anae.15081

PMID: 32311772

2. R. Canelli, C.W. Connor, M. Gonzalez, A. Nozari, R. Ortega. Barrier enclosure during endotracheal intubation. N Engl J Med. 2020; 382 (20): 19571958.

https://doi.org/10.1056/NEJMc2007589

PMID: 32243118

3. Begley J.L., Lavery K.E., Nickson C.P., Brewster D.J. The aerosol box for intubation in coronavirus disease 2019 patients: an in-situ simulation crossover study. Anaesthesia. 2020; 75: 1014-21. https://doi.org/10.1111/anae.15115

PMID: 32397008 